\title{
Characterization of Endophytic Fungi from Western Ghat Orchid : Cleisostoma tenuifolium
}

\author{
Darsha $\mathrm{S}^{*}$, Jayashankar $\mathrm{M}^{2}$
}

${ }^{1}$ Mangalore University, Department of Research and Studies in Microbiology, Mangalore University, PG Centre, Jnana Kaveri Campus, Kodagu, Karnataka, India.

${ }^{2}$ Mangalore University, Department of Research and Studies in Microbiology, Mangalore University, PG Centre, Jnana Kaveri Campus, Kodagu, Karnataka, India

*Corresponding author : darshu11m90@gmail.com

Article Info

Volume 7, Issue 6

Page Number: 33-42

Publication Issue :

November-December-2020

\section{Article History}

Accepted : 01 Nov 2020

Published : 07 Nov 2020

\section{ABSTRACT}

Western Ghats is one of the hottest hot spot in the world. Cleisostoma tenuifolium is an epiphytic orchid from the heart of this region. We have isolated and identified four endophytic fungi from this plant. Characterization of these fungi were done by analyzing the sequences. Genome were quantified and sequenced. Sequence similarity matrix were prepared by comparing the ITS region sequence of obtained fungi with its closely related fungal species from NCBI database. Phylogenetic tree were also constructed for further comparing the homological features of these fungi. From the study, the obtained fungi were Aspergillus flavus MT464452, Cladosporium xanthochromaticum MT464453, Colletotrichum cymbidicola MT464454, Aspergillus aculeatus MT464455. The wide variety of endophytic fungi isolated from the plant $\mathrm{C}$. tenuifolium that belongs to Orchidaceae family further highlights the importance of orchids as reservoirs of fungal biodiversity and its conservation.

Keywords : Western Ghats, Endophytic fungi, Cleisostoma tenuifolium, NCBI, ITS region, Biodiversity

\section{INTRODUCTION}

Orchids are well known for their ornamental and medicinal values, as well as for their features as indicators of ecological integrity. Orchids have been used as food and in traditional medicine for treating several ailments by different cultures and tribes all over the world. Orchids are very sensitive to habitat degradation. Although large population of orchids is confined to their natural habitats, their number is decreasing because of high demand, habitat destruction and indiscriminate collection [9][13]. Cleisostoma tenuifolium is a genus of orchids with approximately 90 accepted species widely distributed through much of Indian subcontinent, Southeast Asia, China, New Guinea and some of the islands of the 
western pacific. Cleisostoma tenuifolium is an epiphytic plant that habitat on tree trunks in broad leaved evergreen forests and semi-evergreen forest. It is a flowering plant with yellow flowers with linear pointed leaves that belongs to the family Orchidaceae which will grow upto 20-30 long [3].

All orchids maintain an obligate relationship with mycorrhizal symbiont [6]. The interaction between orchid and fungi were significantly influenced by the phylogenetic relationship. Mycorrhizal fungi can influence orchid population dynamics [16]. Endophytes are asymptomatic fungi residing in aerial and underground tissues of plants. Endophytes are the subject of bioprospecting exercise owing to their ability to produce novel bioactive compounds [20] and industrial enzymes [19]. These compunds from endophytic fungi find enormous application as agrochemicals, antibiotics, antiparasitics, antioxidants, biopesticides, anticancer agents and also capability to produce extracellular enzymes [14] [15]. Diversity analysis of the associating fungal endophytes is an emerging challenge, which leads to the discovery of new species producing novel compounds and a better understanding of their role in ecosystems [17]. These properties of fungi might be due to the presence of symbiotic endophytes that habitat inside them.

The present study is to identify these fungi to its genotypic level by comparing them with known fungi. Thereby, accessing the diversity of endophytic fungi from epiphytic orchid plant $C$. tenuifolium.

\section{MATERIALS AND METHODS}

\section{Collection of Plant Part}

The plant parts can be randomly cut off with a disinfected sickle and placed separately in sterile polythene bags to avoid moisture loss. The materials were transported to the laboratory within 24 hours and stored at $4^{\circ} \mathrm{C}$ until the isolation procedures were completed from the Somwarpet (75025'-75014'E, $12^{0} 15^{\prime}-12^{0} 45^{\prime} \mathrm{N}$ ) agro-forestry area of Kodagu district ,Karnataka which is the central part of Western Ghats, one of the eight hottest hot spot of biological diversity in the world.

Isolation of endophytes

The plant plants brought into the laboratory were processed within 6hours.The collected plant parts were thoroughly washed in running tap water to remove dirt and debries. Fresh healthy leaves and root were selected for endophyte isolation. Epiphytes were removed from the surface by disinfecting the specimen by $70 \%$ ethanol for 1 minute, $4 \%$ sodium hypochlorite solution for 3minutes; $70 \%$ ethanol for $30 \mathrm{~s}$ and two rinses in sterilized distilled water. After removing the excess water, the leaf and root were excised in to the size of $0.5 \times 0.5 \mathrm{~cm}$ with the help of a sterile blade. A total of 50 segments were screened from each and these segments were placed in containing Potato Dextrose Agar (PDA) amended with chloramphenicol $(150 \mathrm{mg} / \mathrm{L})$. These plates were incubated at room temperature $\left(28^{\circ} \mathrm{C}\right)$ for upto 15 days and were observed periodically for the appearance of fungal colony [10][12]. The efficacy of the sterilization protocol was tested by making an imprint of a surface sterilised segment onto a PDA medium. No fungal colonies grew from these, suggesting that the sterilization protocol was effective [7]. The different mycellium obtained were grown separately on fresh medium and maintained for further experiments.

Isolation of genomic DNA, PCR Amplification and sequencing Quantification of the genomic DNA was done by following the procedure:

The fungus was cultured on the medium potato dextrose agar and the mycelium was suspended in $40 \mu \mathrm{l}$ water, $160 \mu \mathrm{l}$ of $\mathrm{NaOH}$ was added to the culture 
suspension, mixed well and incubated in $60^{\circ} \mathrm{C}$ for 45 minutes. After that $12 \mu \mathrm{l}$ Tris- $\mathrm{HCl}(0.01 \mathrm{M})$ was added and diluted up to 100 fold.the process of PCR was done using the set up:10X PCR buffer:2.5 $\mu \mathrm{l}, \mathrm{MgCl}_{2}: 2 \mu \mathrm{l}$ $(25 \mathrm{mM})$, dNTPs: $5 \mu \mathrm{L}(2 \mathrm{mM})$, Primer $\mathrm{F}$ and primer $\mathrm{R}: 0.5 \mu \mathrm{l}$ each, Taq polymerase $: 0.36 \mu \mathrm{l}(5 \mathrm{U} / \mu \mathrm{l})$, DNA from the diluted extract: $6.0 \mu$. The programme was initiated by denaturation at $95{ }^{\circ} \mathrm{C}$ for 5 minutes.Followed with 35 cycles at $94{ }^{\circ} \mathrm{C}$ for 1 minute, $58{ }^{\circ} \mathrm{C}$ for 1 minute annealing condition and the extention temperature of $72^{\circ} \mathrm{C}$ for 1 minute in 35 cycles and final extension at $72^{\circ} \mathrm{C}$ for 5 minutes and hold at $4{ }^{\circ} \mathrm{C}$ for infinite time. After PCR, the product was processed through gel electrophoresis and then sequenced. The similarity of the sequence was compared with known culture from NCBI data base. The similarity matrix was generated by using the BioEdit 7.0.4.1 software [5].

\section{Phylogenetic analysis}

Phylogenetic tree is constructed by comparing with strains from GenBank with highest similarities (accession numbers in parentheses). Tree is constructed using kimura 2 model with bootstrap method. The neighbor-joining tree and subtree were generated using MEGA5.2 software [11].

\section{III.RESULTS}

After collecting the root sample from the epiphytic orchid, $C$ tenuifolium a number of fungal cultures were obtained. Only a few selected ones were proceeded for genomic analysis. Four endophytic fungi were selected from the samples and named the fungi BR1, BR2, BR3 and BR4 for our convenience. The isolated genomic DNA of these endophytic fungi were amplified by PCR program and ITS region were sequenced. Fungi that are closely related and the ITS region sequence obtained were analysed for its similarity. The sequences obtained were submitted to Genbank for Accession numbers; submission code and the accession number of each bacteria were given in the Table 1.From the information obtained, similarity matrix of each fungi were prepared and tabulated as: Table 2, 3,4 and 5. For knowing the fungal history phylogenetic tree were generated and were mentioned in the Figure 1, 2, 3, and 4.

Table 1 : showing submission code and accession number for corresponding sequences

\begin{tabular}{|l|l|l|l|}
\hline $\begin{array}{l}\text { Sl. } \\
\text { No. }\end{array}$ & Fungi & $\begin{array}{l}\text { Submission } \\
\text { code }\end{array}$ & Accession number \\
\hline 1 & Aspergillus flavus & SUB7444841 & MT464452 \\
\hline 2 & Cladosporium xanthochromaticum & SUB7444841 & MT464453 \\
\hline 3 & Colletotrichum cymbidicola & SUB7444841 & MT464454 \\
\hline 4 & Aspergillus aculeatus & SUB7444841 & MT464455 \\
\hline
\end{tabular}

Based on the experimental results, the fungal culture has been identified as: BR1: According to NCBI data base, sequence is having highest similarity with Aspergillus sp. Following BLASTn search and sequence alignment the sequence is have $100 \%$ similarity with Aspergillus flavus. BR2: According to NCBI data base, sequence is having highest similarity with Cladosporium $s p$. Following BLASTn search and sequence alignment the sequence is have $100 \%$ match with Cladosporium xanthochromaticum and Cladosporium perangustum. BR3: According to NCBI data base, sequence is having highest similarity with Colletotrichum sp. Following BLASTn search and sequence alignment the sequence is have $100 \%$ similarity with Colletotrichum cymbidicola. BR4: 
According to NCBI data base, sequence is having highest similarity with Aspergillus sp. Following BLASTn search and sequence alignment the sequence is have 100\% similarity with Aspergillus aculeatus and 99.82\% with Aspergillus japonicus. Briefly the fungi were Aspergillus flavus, Cladosporium xanthochromaticum, Colletotrichum cymbidicola, Aspergillus aculeatus.

Table 2 : Sequence similarity matrix of BR1- ITS region with the other closely related species

\begin{tabular}{|l|l|l|l|l|l|l|l|l|l|l|}
\hline Sequence & 1 & 2 & 3 & 4 & 5 & 6 & 7 & 8 & 9 & 10 \\
\hline BR1 & - & & & & & & & & & \\
\hline $\begin{array}{l}\text { A_nomius_voucher_NWUSeq52_(MK841463. } \\
\text { 1) }\end{array}$ & 100 & - & & & & & & & & \\
\hline A_flavus_voucher_NWUSeq49_(MK841461.1) & 100 & 100 & - & & & & & & & \\
\hline Aspergillus_sp_isolate_SFS-6_(MK817596.1) & 100 & 100 & 100 & - & & & & & & \\
\hline A_flavus_isolate_8_(MK494007.1) & 100 & 100 & 100 & 100 & - & & & & & \\
\hline A_flavus_isolate_196_(MK493836.1) & 100 & 100 & 100 & 100 & 100 & - & & & & \\
\hline A_flavusisolate_215_(MK493817.1) & 100 & 100 & 100 & 100 & 100 & 100 & - & & & \\
\hline $\begin{array}{l}\text { C_fructicola_culture_ICMP:18581_(JX010165. } \\
\text { 1) }\end{array}$ & 69.1 & 69.1 & 69.1 & 69.1 & 69.1 & 69.1 & 69.1 & - & & \\
\hline A_flavus_A581_(KX463034.1) & 98.4 & 98.4 & 98.4 & 98.4 & 98.4 & 98.4 & 98.4 & 68.1 & - & \\
\hline Fusarium_sp_TMS-2011_(HQ631016.1) & 65.2 & 65.2 & 65.2 & 65.2 & 65.2 & 65.2 & 65.2 & 76.3 & 64 & - \\
\hline
\end{tabular}

Table 3 : Sequence similarity matrix of BR2- ITS region with the other closely related species

\begin{tabular}{|l|l|l|l|l|l|l|l|l|l|l|l|}
\hline Sequence & 1 & 2 & 3 & 4 & 5 & 6 & 7 & 8 & 9 & 10 & 11 \\
\hline BR2 & - & & & & & & & & & & \\
\hline $\begin{array}{l}\text { Cladosporium_sp_strain_APBSDSF50_(MG66 } \\
\text { 9182.1) }\end{array}$ & 100 & - & & & & & & & & & \\
\hline $\begin{array}{l}\text { C_xanthochromaticum_DTO:323- } \\
\text { E6_(MF473322.1) }\end{array}$ & 100 & 100 & - & & & & & & & & \\
\hline C_perangustum_CPC:18496_(KT600414.1) & $\begin{array}{l}99 . \\
8\end{array}$ & $\begin{array}{l}99 . \\
8\end{array}$ & $\begin{array}{l}99 . \\
8\end{array}$ & - & & & & & & & \\
\hline C_perangustum_isolate_Gua1_(JN698599.1) & $\begin{array}{l}99 . \\
8\end{array}$ & $\begin{array}{l}99 . \\
8\end{array}$ & $\begin{array}{l}99 . \\
8\end{array}$ & 100 & - & & & & & & \\
\hline $\begin{array}{l}\text { C_angustisporum_strain_CBS125983_(MH86 } \\
\text { 3862.1) }\end{array}$ & $\begin{array}{l}99 . \\
6\end{array}$ & $\begin{array}{l}99 . \\
6\end{array}$ & $\begin{array}{l}99 . \\
6\end{array}$ & $\begin{array}{l}99 . \\
8\end{array}$ & $\begin{array}{l}99 . \\
8\end{array}$ & - & & & & & \\
\hline $\begin{array}{l}\text { C_cladosporioides_isolate_JRBP2015.838b_ } \\
\text { (MH399496. 1) }\end{array}$ & $\begin{array}{l}99 . \\
6\end{array}$ & $\begin{array}{l}99 . \\
6\end{array}$ & $\begin{array}{l}99 . \\
6\end{array}$ & $\begin{array}{l}99 . \\
8\end{array}$ & $\begin{array}{l}99 . \\
8\end{array}$ & 100 & - & & & & \\
\hline $\begin{array}{l}\text { C_perangustum_culture_CPC:22378_(MF473 } \\
\text { 178.1) }\end{array}$ & $\begin{array}{l}99 . \\
6\end{array}$ & $\begin{array}{l}99 . \\
6\end{array}$ & $\begin{array}{l}99 . \\
6\end{array}$ & $\begin{array}{l}99 . \\
8\end{array}$ & $\begin{array}{l}99 . \\
8\end{array}$ & 100 & 100 & - & & & \\
\hline $\begin{array}{l}\text { C_fructicola_culture_ICMP:18581_(JX010165 } \\
\text {.1) }\end{array}$ & $\begin{array}{l}71 . \\
4\end{array}$ & $\begin{array}{l}71 . \\
4\end{array}$ & $\begin{array}{l}71 . \\
4\end{array}$ & $\begin{array}{l}71 . \\
6\end{array}$ & $\begin{array}{l}71 . \\
6\end{array}$ & 71. & 71. & 71. & - & & \\
\hline
\end{tabular}




\begin{tabular}{|l|l|l|l|l|l|l|l|l|l|l|l|}
\hline A_flavus_A581_(KX463034.1) & 67. & 67. & 67. & 66. & 66. & 67. & 67. & 67. & 63. & - & \\
1 & 1 & 1 & 9 & 9 & 1 & 1 & 1 & 5 & \\
\hline Fusarium_sp_TMS-2011_(HQ631016.1) & 72 & 72 & 72 & 72 & 72 & $\begin{array}{l}72 . \\
2\end{array}$ & $\begin{array}{l}72 . \\
2\end{array}$ & $\begin{array}{l}72 . \\
2\end{array}$ & $\begin{array}{l}77 . \\
2\end{array}$ & $\begin{array}{l}61 . \\
3\end{array}$ \\
\hline
\end{tabular}

Table 4 : Sequence similarity matrix of BR3- ITS region with the other closely related species

\begin{tabular}{|c|c|c|c|c|c|c|c|c|c|c|c|}
\hline Sequence & 1 & 2 & 3 & 4 & 5 & 6 & 7 & 8 & 9 & 10 & 11 \\
\hline$B R 3$ & - & & & & & & & & & & \\
\hline $\begin{array}{l}\text { Colletotrichum_sp_isolate_A934_(MK24762 } \\
\text { 8.1) }\end{array}$ & 100 & - & & & & & & & & & \\
\hline $\begin{array}{l}\text { C_cymbidiicola_strain_CBS_128543_(MH86 } \\
\text { 5014.1) }\end{array}$ & 100 & 100 & - & & & & & & & & \\
\hline C_cymbidiicola_strain_JL-3_(KX058529.1) & 100 & 100 & 100 & - & & & & & & & \\
\hline $\begin{array}{l}\text { C_boninense_culture_ICMP:17904_(JX01029 } \\
\text { 2.1) }\end{array}$ & $\begin{array}{l}99 . \\
6\end{array}$ & $\begin{array}{l}99 . \\
6\end{array}$ & $\begin{array}{l}99 . \\
6\end{array}$ & $\begin{array}{l}99 . \\
6\end{array}$ & - & & & & & & \\
\hline $\begin{array}{l}\text { Colletotrichum_sp_ICMP17319_(EU482213. } \\
\text { 1) }\end{array}$ & $\begin{array}{l}99 . \\
6\end{array}$ & $\begin{array}{l}99 . \\
6\end{array}$ & $\begin{array}{l}99 . \\
6\end{array}$ & $\begin{array}{l}99 . \\
6\end{array}$ & $\begin{array}{l}99 . \\
2\end{array}$ & - & & & & & \\
\hline C_boninense_strain_AR3751_(DQ286170.1) & $\begin{array}{l}98 . \\
6\end{array}$ & $\begin{array}{l}98 . \\
6\end{array}$ & $\begin{array}{l}98 . \\
6\end{array}$ & $\begin{array}{l}98 . \\
6\end{array}$ & $\begin{array}{l}98 . \\
6\end{array}$ & $\begin{array}{l}98 . \\
2\end{array}$ & - & & & & \\
\hline $\begin{array}{l}\text { Colletotrichum_sp_1WCC- } \\
\text { 2019_NTOU:4895_ } \\
(M K 432993.1)\end{array}$ & $\begin{array}{l}96 . \\
9\end{array}$ & $\begin{array}{l}96 . \\
9\end{array}$ & $\begin{array}{l}96 . \\
9\end{array}$ & $\begin{array}{l}96 . \\
9\end{array}$ & $\begin{array}{l}96 . \\
5\end{array}$ & $\begin{array}{l}96 . \\
5\end{array}$ & $\begin{array}{l}95 . \\
6\end{array}$ & - & & & \\
\hline $\begin{array}{l}\text { C_fructicola_culture_ICMP:18581_(JX01016 } \\
\text { 5.1) }\end{array}$ & $\begin{array}{l}92 . \\
4\end{array}$ & $\begin{array}{l}92 . \\
4\end{array}$ & $\begin{array}{l}92 . \\
4\end{array}$ & $\begin{array}{l}92 . \\
4\end{array}$ & $\begin{array}{l}92 . \\
2\end{array}$ & 92 & 92 & $\begin{array}{l}89 . \\
3\end{array}$ & - & & \\
\hline A_flavus_A581_(KX463034.1) & $\begin{array}{l}64 . \\
7\end{array}$ & $\begin{array}{l}64 . \\
7\end{array}$ & $\begin{array}{l}64 . \\
7\end{array}$ & $\begin{array}{l}64 . \\
7\end{array}$ & $\begin{array}{l}64 . \\
8\end{array}$ & $\begin{array}{l}64 . \\
5\end{array}$ & $\begin{array}{l}64 . \\
3\end{array}$ & $\begin{array}{l}63 . \\
9\end{array}$ & $\begin{array}{l}63 . \\
7\end{array}$ & - & \\
\hline Fusarium_sp_TMS-2011_(HQ631016.1) & $\begin{array}{l}74 . \\
1\end{array}$ & $\begin{array}{l}74 . \\
1\end{array}$ & $\begin{array}{l}74 . \\
1\end{array}$ & $\begin{array}{l}74 . \\
1\end{array}$ & $\begin{array}{l}74 . \\
1\end{array}$ & $\begin{array}{l}74 . \\
1\end{array}$ & $\begin{array}{l}73 . \\
9\end{array}$ & $\begin{array}{l}71 . \\
1\end{array}$ & $\begin{array}{l}74 . \\
4\end{array}$ & $\begin{array}{l}57 . \\
7\end{array}$ & - \\
\hline
\end{tabular}

Table 5 : Sequence similarity matrix of BR4- ITS region with the other closely related species

\begin{tabular}{|l|l|l|l|l|l|l|l|l|l|l|l|l|l|}
\hline Sequence & 1 & 2 & 3 & 4 & 5 & 6 & 7 & 8 & 9 & 10 & 11 & 12 & 13 \\
\hline BR4 & - & & & & & & & & & & & & \\
\hline $\begin{array}{l}\text { A_aculeatus_strain_JOF07_ } \\
\text { (MG589506.1) }\end{array}$ & $\begin{array}{l}96 . \\
3\end{array}$ & - & & & & & & & & & & & \\
\hline $\begin{array}{l}\text { A_japonicus_strain_SK1_ } \\
\text { (KY199566.1) }\end{array}$ & $\begin{array}{l}97 . \\
4\end{array}$ & $\begin{array}{l}93 . \\
9\end{array}$ & - & & & & & & & & & & \\
\hline $\begin{array}{l}\text { A_aculeatus_strain_HHNP- } \\
\text { FS01_ } \\
\text { (KR296866.1) }\end{array}$ & $\begin{array}{l}96 . \\
1\end{array}$ & $\begin{array}{l}92 . \\
6\end{array}$ & $\begin{array}{l}97 . \\
2\end{array}$ & - & & & & & & & & & \\
\hline
\end{tabular}




\begin{tabular}{|c|c|c|c|c|c|c|c|c|c|c|c|c|}
\hline $\begin{array}{l}\text { A_japonicus_strain_VIT- } \\
\text { SB1_(KC128815.1) }\end{array}$ & $\begin{array}{l}99 . \\
8\end{array}$ & $\begin{array}{l}96 . \\
5\end{array}$ & $\begin{array}{l}97 . \\
4\end{array}$ & $\begin{array}{l}96 . \\
1\end{array}$ & - & & & & & & & \\
\hline $\begin{array}{l}\text { A_aculeatus_isolate_A3S1_D57 } \\
- \\
\text { (JX501397.1) }\end{array}$ & 99 & $\begin{array}{l}95 . \\
7\end{array}$ & $\begin{array}{l}98 . \\
1\end{array}$ & $\begin{array}{l}96 . \\
8\end{array}$ & $\begin{array}{l}99 . \\
2\end{array}$ & - & & & & & & \\
\hline $\begin{array}{l}\text { A_aculeatus_isolate_F36_ } \\
\text { (JF439460.1) }\end{array}$ & 100 & $\begin{array}{l}96 . \\
3\end{array}$ & $\begin{array}{l}97 . \\
4\end{array}$ & $\begin{array}{l}96 . \\
1\end{array}$ & $\begin{array}{l}99 . \\
8\end{array}$ & 99 & - & & & & & \\
\hline $\begin{array}{l}\text { A_japonicus_strain_MUCL1357 } \\
\text { 8_ } \\
(\text { AJ876880.1) }\end{array}$ & $\begin{array}{l}98 . \\
9\end{array}$ & $\begin{array}{l}95 . \\
6\end{array}$ & $\begin{array}{l}98 . \\
3\end{array}$ & 97 & 99 & $\begin{array}{l}99 . \\
8\end{array}$ & $\begin{array}{l}98 . \\
9\end{array}$ & - & & & & \\
\hline $\begin{array}{l}\text { A_aculeatus_strain_CBS17266_ } \\
\text { (AY585558.1) }\end{array}$ & $\begin{array}{l}95 . \\
6\end{array}$ & $\begin{array}{l}92 . \\
1\end{array}$ & $\begin{array}{l}96 . \\
6\end{array}$ & $\begin{array}{l}99 . \\
4\end{array}$ & $\begin{array}{l}95 . \\
6\end{array}$ & $\begin{array}{l}96 . \\
3\end{array}$ & $\begin{array}{l}95 . \\
6\end{array}$ & $\begin{array}{l}96 . \\
4\end{array}$ & - & & & \\
\hline $\begin{array}{l}\text { A_aculeatus_isolate_NRRL5094 } \\
- \\
\text { (EF661221.1) }\end{array}$ & $\begin{array}{l}93 . \\
4\end{array}$ & $\begin{array}{l}89 . \\
9\end{array}$ & $\begin{array}{l}95 . \\
5\end{array}$ & $\begin{array}{l}97 . \\
1\end{array}$ & $\begin{array}{l}93 . \\
4\end{array}$ & $\begin{array}{l}94 . \\
1\end{array}$ & $\begin{array}{l}93 . \\
4\end{array}$ & $\begin{array}{l}94 . \\
2\end{array}$ & $\begin{array}{l}97 . \\
7\end{array}$ & - & & \\
\hline $\begin{array}{l}\text { C_fructicola_culture_ICMP:185 } \\
81 \_ \\
\text {(JX010165.1) }\end{array}$ & $\begin{array}{l}68 . \\
7\end{array}$ & $\begin{array}{l}65 . \\
7\end{array}$ & $\begin{array}{l}66 . \\
7\end{array}$ & $\begin{array}{l}65 . \\
7\end{array}$ & $\begin{array}{l}68 . \\
8\end{array}$ & $\begin{array}{l}68 . \\
1\end{array}$ & $\begin{array}{l}68 . \\
7\end{array}$ & $\begin{array}{l}68 . \\
1\end{array}$ & $\begin{array}{l}65 . \\
2\end{array}$ & $\begin{array}{l}63 . \\
2\end{array}$ & - & \\
\hline A_flavus_A581_(KX463034.1) & $\begin{array}{l}83 . \\
8\end{array}$ & $\begin{array}{l}82 . \\
1\end{array}$ & $\begin{array}{l}83 . \\
1\end{array}$ & $\begin{array}{l}81 . \\
9\end{array}$ & 84 & $\begin{array}{l}84 . \\
6\end{array}$ & $\begin{array}{l}83 . \\
8\end{array}$ & $\begin{array}{l}84 . \\
7\end{array}$ & $\begin{array}{l}81 . \\
3\end{array}$ & $\begin{array}{l}79 . \\
2\end{array}$ & $\begin{array}{l}65 . \\
3\end{array}$ & - \\
\hline $\begin{array}{l}\text { Fusarium_sp_TMS-2011_ } \\
(\text { HQ631016.1) }\end{array}$ & $\begin{array}{l}66 . \\
9\end{array}$ & $\begin{array}{l}63 . \\
9\end{array}$ & $\begin{array}{l}64 . \\
9\end{array}$ & $\begin{array}{l}63 . \\
9\end{array}$ & 67 & $\begin{array}{l}66 . \\
3\end{array}$ & $\begin{array}{l}66 . \\
9\end{array}$ & $\begin{array}{l}66 . \\
3\end{array}$ & $\begin{array}{l}63 . \\
3\end{array}$ & $\begin{array}{l}61 . \\
4\end{array}$ & $\begin{array}{l}77 . \\
4\end{array}$ & $\begin{array}{l}61 . \\
7\end{array}$ \\
\hline
\end{tabular}

Given below is the figure 1 indicating phylogenetic tree of fungal culture BR1

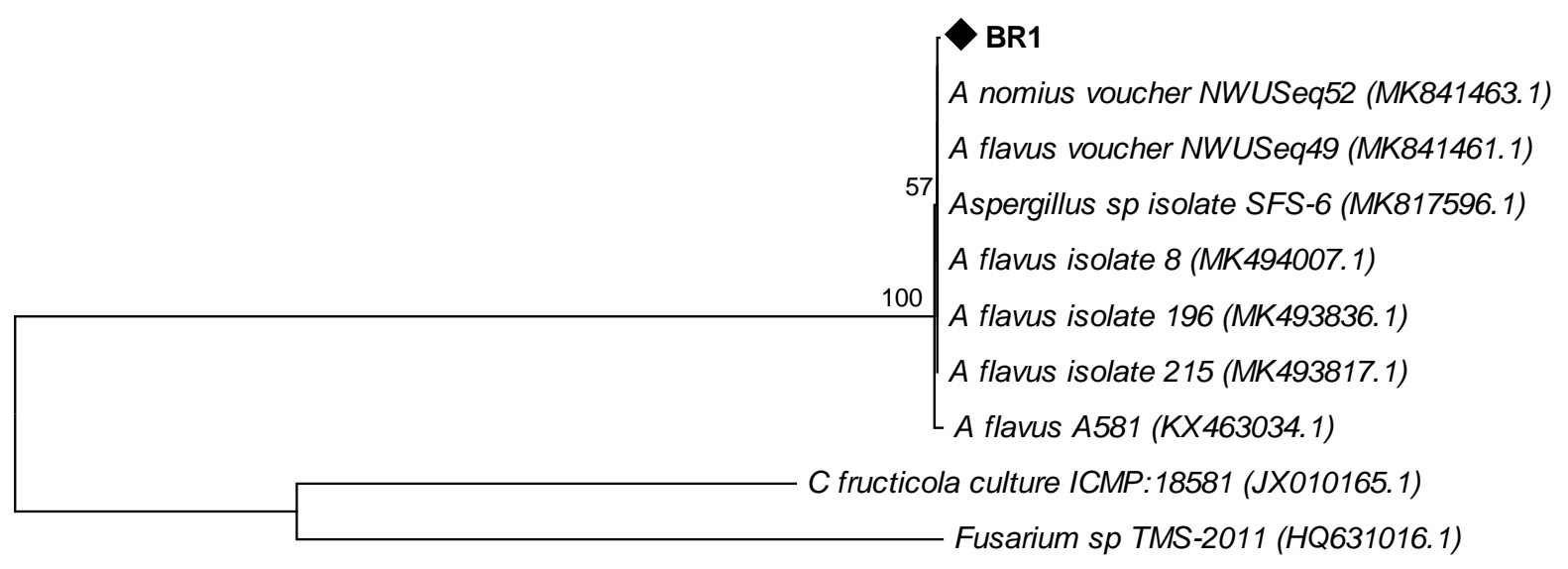




\section{Given below is the figure 2 indicating phylogenetic tree of fungal culture BR2}

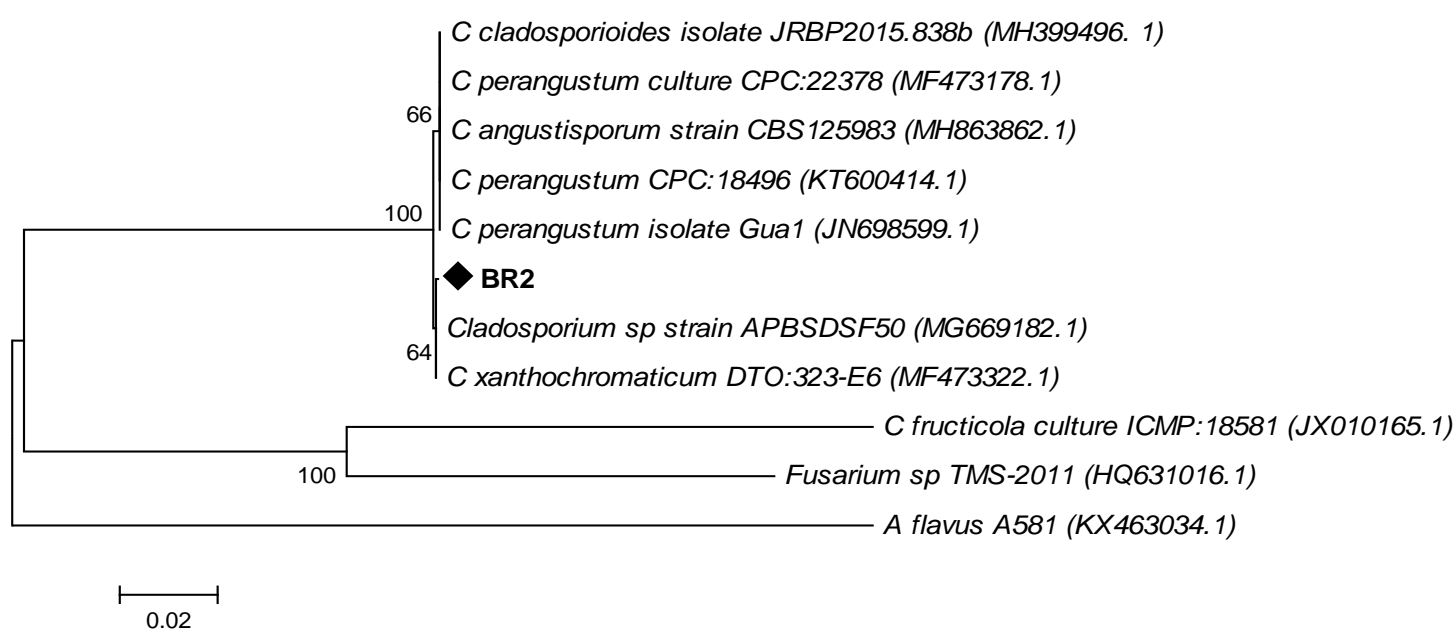

Given below is the figure 3 indicating phylogenetic tree of fungal culture BR3

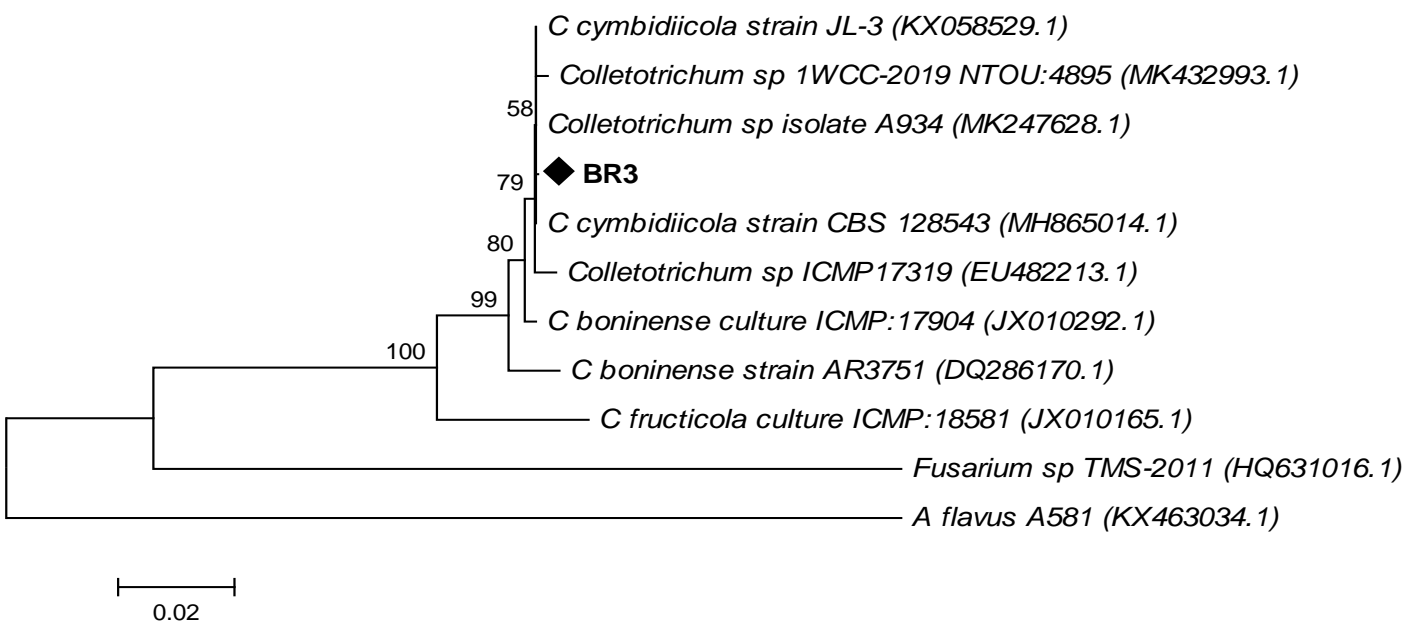

Given below is the figure 4 indicating phylogenetic tree of fungal culture BR4

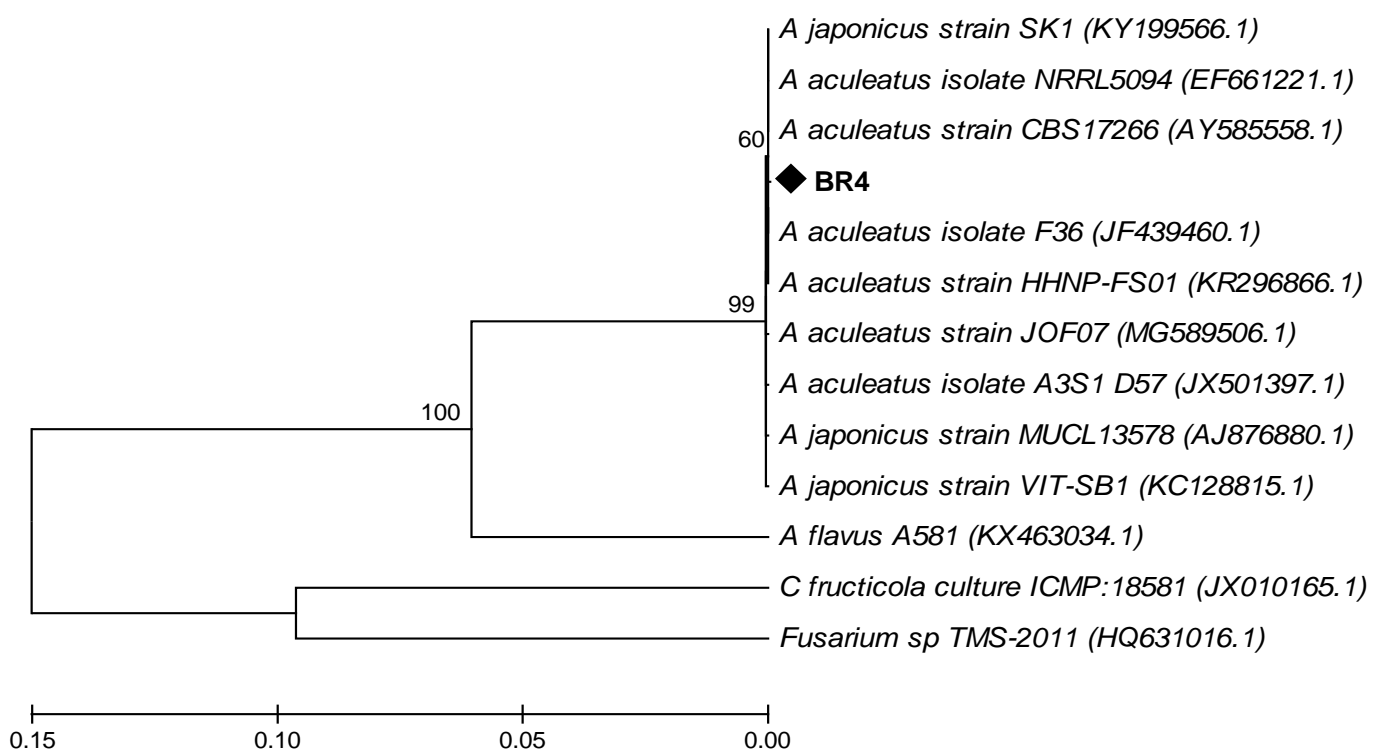




\section{Discussion}

All orchids maintain an obligate relationship with mycorrhizal symbiont [6]. The interaction between orchid and fungi were significantly influenced by the phylogenetic relationship. Mycorrhizal fungi can influence orchid population dynamics [16]. Orchid species are critically dependent on mycorrhizal fungi for completion of their life cycle, particularly during the early stages of their development when nutritional resources are scarce. Mycorrhizal specificity was low, but significant variation in mycorrhizal community composition was observed between species inhabiting different ecological habitats. Molecular identification of endophytic fungi from the epiphytic fungi Vanda testacea were discussed in our previos study [2].

Although several fungi had a broad geographic distribution, Species Indicator Analysis revealed some fungi that were characteristic for specific habitats. Overall, these results indicate that orchid mycorrhizal fungi may have a broad geographic distribution, but that their occurrence is bounded by specific habitat conditions [8]. Kotain et al [10] found endophytes were shared by the two different orchids growing in the same location when compared to endophyte assemblages of a single orchid from different locations.

Considering the influence of endophytes in shaping the host's community, diverse habitats must be screened vigorously to address questions regarding the role of endophytes in host-endophyte interactions. The pioneer studies of orchid endophytic fungi have been conducted by Warcup and Talbot and worked with isolates from Australian terrestrial orchids [1]. Only a few studies were conducted in relation with orchid-endophytes. Non-mycorrhizal fungal endophytes in two orchids of kaiga forest [18]. Fungal endophytes from two orchid species-pointer towards organ specificity [10]. The microbial advantage in drug discovery is profound, offering several distinct advantages. The first is the negligible impact to the environment incurred in the collection process. Each microbe can produce metabolites with desirable bioactivity.

Significantly, many of these studies have used traditional morphological methods to identify isolated endophytes which raises the possibility that some fungi may have been misidentified or overlooked [4]. Here, we identified four endophytic fungi up to its genomic level from the root of a single orchid. It is best to use molecular sequencing methods for identifying fungi than by observing its morphology, since it provide a vast knowledge upto its species level. To the best of our knowledge, this is the first study in account of molecularly identifying endophytic fungi (Aspergillus flavus, Cladosporium xanthochromaticum, Colletotrichum cymbidicola, Aspergillus aculeatus) from the epiphytic orchid, $C$. tenuifolium, generating similarity matrix and also comparing those fungi with similar ones by constructing phylogenetic tree.

\section{Conclusion}

Briefly, we have documented a range of fungi from a small plant sample from one of the eight hottest hot spot (Western Ghats) in the World. In this study, we have characterized endophytic fungi from the epiphytic orchid plant $C$ tenuifolium to its genotypic level. Such a diversified variety of fungi illustrates the importance of conserving plant reservoirs and fungal biodiversity. This study provides propulsion for future studies related to orchids and endophytes. 


\section{Acknowledgement}

We would like to thank Mangalore University for its collaboration and providing facilities for the study.

\section{VII.REFERENCES}

[1]. Currah RS, Sigler L, Hambleton S.1987. New records and new taxa of fungi from the rnycorrhizae of terrestrial orchids of Alberta. Canadian Journal of Botany, 65(12): 2473-2482. https://doi.org/10.1139/b87-336

[2]. Darsha S and Jayashankar M. 2020. Molecular characterization of bacterial and fungal endophytes associat-ed with Vanda testacea, an orchid of Kodagu forest (Western Ghats), India. South Asian J Experim Bio, 10(5): 292-300

[3]. Gogoi K, Borah RL, Das R, Yonzone R. 2012. Present Status of Orchid Species Diversity Resources of Joypur Reserve Forest of Dibrugarh District (Assam) of North East India Intern Jour of Modern Botany, 2(3): 47-67. https://doi.org/10.5923/j.ijmb.20120203.03

[4]. Guo LD, Hyde KD and Edward CYL.2001. Detection and taxonomic placement of endophytic fungi within frond tissues of Livistonachinensis based on rDNA sequences. Mol.Phylogenet. Evol, 20(1): 1-13. https://doi.org/10.1006/mpev.2001.0942

[5]. Hall TA.1999. BioEdit: a user-friendly biological sequence alignment editor and analysis program for Windows 95/98/NT. Nucl. Acids. Symp. Ser, 41:95-98.

[6]. Huang H, Zi XM, Lin H, Gao JY.2018. Hostspecificity of symbiotic mycorrhizal fungi for enhancing seed germination, protocorm formation and seedling development of overcollected medicinal orchid, Dendrobium devonianum. Journal of Microbiology, 56(1):4248. https://doi.org/10.1007/s12275-018-7225-1
[7]. Hyde KD and Soytong K.2008. The fungal endophyte dilemma. Fungal Divers, 33:163-173.

[8]. Jacquemyn H, Waud M, Vincent SF, Merckx T, Brys R, Tyteca D, Hedrén M, Lievens B.2016. Habitat-driven variation in mycorrhizal communities in the terrestrial orchid genus Dactylorhiza. Scientific Reports, 6(1). https://doi.org/10.1038/srep37182.

[9]. Jalal JS and Jayanthi J.2012. Endemic orchids of peninsular India: a review. Journal of Threatened Taxa, 4(15): 3415-3425. https://doi.org/10.11609/jott.o3091.3415-25

[10]. Kotain S, Vasudevan TG, Murali TS.2013. Fungal endophytes from two orchid species pointer towards organ specificity. Czech Mycology, 65(1):89-101. https://doi.org/10.33585/cmy.65107

[11]. Kumar S, Nei M, Dudley J and Tamura K. 2008. Mega: a biologist-centric software for evolutionary analysis of DNA and protein sequences. Briefings in Bioinformatics, 9(4): 299-306 https://doi.org/10.1093/bib/bbn017

[12]. Nalini MS, Sunayana N and Prakash HS.2014. Endophytic Fungal Diversity in Medicinal Plants of Western Ghats, India. International Journal of Biodiversity, 2014: 1-9. https://doi.org/10.1155/2014/494293

[13]. Pankaj K, Jalal JS, Singh G, Kumar RP, Jalal JS, Rawat GS.2007.Orchidaceae, Chotanagpur, state of Jharkhand, India. Check List, 3(4):297304. https://doi.org/10.15560/3.4.297

[14]. Pavithra NL, Sathish K, Ananda K. 2012. Antimicrobial and enzyme activity of endophytic fungi isolated from Tulsi. J Pharm Biomed Sci.16.

[15]. Pinheiro EA, Carvalho JM, Santos DC, Ade OF, Marinho PS, Guilhon GM. 2013. Antibacterial activity of alkaloids produced by endophytic fungus Aspergillus sp. EJC08 isolated from medical plant Bauhinia guianensis. Natural 
Product Research, 27(18):1633-1638. Cite this article as :

https://doi.org/10.1080/14786419.2012.750316

[16]. Rock-Blake R, McCormick MK, Brooks HEA, Cynyhia SJ and Dennis FW.2017. Symbiont

Darsha S, Jayashankar M, "Characterization of abundance can affect host plant population dynamics. American Journal of Botany, 104(1):72-82.

https://doi.org/10.3732/ajb.1600334

[17]. Rodriguez RJ, White JF, Arnold AE, Redman RS. 2009. Fungal endophytes: diversity and functional roles. New Phytologist, 182(2):314Endophytic fungi from Western Ghat orchid: Cleisostoma tenuifolium ", International Journal of Scientific Research in Science and Technology (IJSRST), Online ISSN : 2395-602X, Print ISSN : 23956011, Volume 7 Issue 6, pp. 33-42, NovemberDecember 2020. Available at doi $\quad$ : https://doi.org/10.32628/IJSRST20765 $30 . \quad$ https://doi.org/10.1111/j.1469Journal URL : http://ijsrst.com/IJSRST20765

8137.2009.02773.x

[18]. Sudheep NM and Sridhar KR. 2012. Nonmycorrhizal fungal endophytes in two orchids of Kaiga forest (Western Ghats), India. Journal of Forestry Research, 23(3): 453-460. https://doi.org/10.100/s11676-0120284-y

[19]. Suryanarayanan TS, Thirunavukkarasu N, Rajulu GMB, Gopalan V.2012. Fungal endophytes: an untapped source of biocatalysts. Fungal Diversity, 54(1): 19-30. https://doi.org/10.1007/s13225-012-0168-7

[20]. Suryanarayanan TS, Thirunavukkarasu N, Govindarajulu MB, Sasse F, Jansen R, Murali TS. 2009. Fungal endophytes and bioprospecting. Fungal Biology Reviews, 23(12):

https://doi.org/10.1016/j.fbr.2009.07.001 